\title{
Particle Acceleration during Counterstreaming Instability in Magnetized Pair Plasmas
}

\author{
Laboratory for Plasma Astrophysics, Faculty of Engineering \\ Shinji Saito and Jun-ichi Sakai \\ d023008@ems.toyama-u.ac.jp
}

\begin{abstract}
The electromagnetic counterstreaming instability in unmagnetized pair (electron-positron) plasmas which is influenced by the external magnetic field parallel to the streaming direction is investigated numerically. The simulation show that the counterstreaming instability changes its character from magnetic to electrostatic nature. The electrostatic waves growing due to the electrostatic counterstreaming instability play an important role for producing fast electrons and positrons with energy of $\mathrm{MeV}$. The process of high-energy particle production in relativistic shocks in magnetized pair plasmas may be applied to gamma-ray burst events.

Keywords : particle acceleration, counterstreaming, pair plasma
\end{abstract}

\section{Introduction}

Gamma-ray bursts (GRBs)[1] are the most powerful events in nature. These events release most of energy as photons with energies in the range from $30 \mathrm{keV}$ to a few $\mathrm{MeV}$. The data are in general agreement with a relativistic shock model, where the prompt and afterglow emissions correspond to synchrotron radiation from shock-accelerated electrons. Therefore, the existence of magnetic fields in the relativistic shock model of the GRBs is essential to explain the emission by the synchrotron radiation [2]. Medvedev and Loeb [3] showed through a linear kinetic treatment that a two stream magnetic instability - a generalization of the Weibel instability [4] - can naturally generate strong magnetic fields in collisionless shock fronts. The recent discovery of a strongly polarized GRBs [5] support the presence of strong, ordered magnetic field at the GRBs source. However, since there is only single observation for it, more evidence may be required to demonstrate the existence of ordered magnetic field.

This two stream magnetic instability has a character similar to Weibel instability[4] that occurs in the plasma with anisotropic temperature. Then this instability is referred to as 'Weibel-type instability' or 'two stream magnetic instability', or 'electromagnetic counterstreaming instability' (EMCSI). This instability is also well known in laserplasma interactions[6][7][8]. Sakai et al.[8]and Saito et al. [9] investigated both analytically and numerically the electron-electron counterstreaming instability in laser plasma by using both two-fluids equations with Maxwell equations and particle-in-cell (PIC) simulations. They showed that in the nonlinear stage of the instability there occurs the for- mation of large-scale, long living electron vortices associated with strong magnetic fields.

Kazimura et al.[10] applied this instability to the collision of pulsar winds. They solved the linear theory with four-fluids (contains both electron and positron fluid) model which is expanded from two-fluids model described by Califano et al.[6]. They found from the linear theory that the quasistatic magnetic fields are generated by the instability. Further, they presented the results of twodimensional PIC simulation of collision of electronpositron plasma clouds. It was shown from the simulation that the magnetic fields with large scale, long living structures are generated and high-energy particles are produced in relativistic collisionless shock fronts. Haruki et al.[11] also investigated this problem with longer time development. They found the production of high-energy particles due to electrostatic waves in the non-linear phase of counterstreaming instability.

Recently the counterstreaming instability has been investigated by using three dimensional PIC simulations[12] [13] [14]. They showed that the generated quasi-static magnetic fields evolve from the short-scale to long-scale magnetic field. Only Nishikawa et al.[12] investigated the effects of weak external magnetic field to counterstreaming instability. Their result was similar to those without the external magnetic field. However, the nature of counterstreaming instability may be affected by the relatively strong external magnetic field, because the generation of the magnetic field is related to the electric currents created by the fluctuating particles motion related to the magnetic fields.

In this paper we investigate numerically, how the counterstreaming instability in unmagnetized pair 


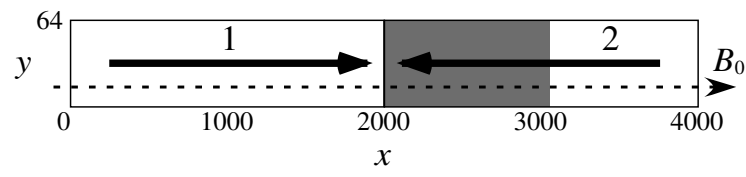

Figure 1: The schematic picture of our simulation model. The notation 1 and 2 mean two counterstreaming components, and the dashed arrow shows the external magnetic field $B_{0}$. The physical processes in the dark region are mainly analyzed.

plasmas is influenced by the external magnetic field parallel to the streaming direction. We show that the EM-CSI changes its character from magnetic to electrostatic nature. The electrostatic waves growing due to the electrostatic counterstreaming instability (ES-CSI) play an important role for producing fast electrons and positrons with energy of $\mathrm{MeV}$. The process of high-energy particle production in relativistic shocks in magnetized pair plasmas may be applied to GRBs event.

\section{Simulation Model}

We used 2D3V, fully relativistic electromagnetic PIC code, modified from 3D3V TRISTAN code [15]. As shown in Fig.1, the system size is $L_{x}=4000 \Delta$ and $L_{y}=64 \Delta$, where $\Delta(=1$.) is grid size. The periodic boundary conditions for both $\mathrm{x}$ and $\mathrm{y}$ directions are imposed on particles and fields. There are about 15 million electron-positron pairs, uniformly and keeping the charge neutrality in the system. The average numbers of electron-positron pairs is about 60 per cell. The initial state of the plasma in the left side region $x \leq 2000 \Delta$ has the shifted Maxwellian with $v_{d}=+0.5 c$, and in the right side region $x>2000 \Delta$ has that with the $v_{d}=-0.5 c$, where $\mathrm{c}$ is light velocity. This drift velocity parallel to the $\mathrm{x}$-direction corresponds to a Lorentz factor $\Gamma=\left[1-\left(v_{d} / c\right)^{2}\right]^{-1 / 2}=1.15$. The thermal velocity of both electron and positron in whole region is $0.1 \mathrm{c}$. Other parameters are as follows : the time step $\omega_{p e} \Delta t=0.05$, mass ratio $m_{p} / m_{e}=1$., Debye length $v_{t h, e} / \omega_{p e}=1$., the collisionless skin depth $c / \omega_{p e}=10 \Delta$. The external magnetic field is parallel to the counterstreaming direction (x-direction), and the initial electric field and electric current are equal to zero. We compare the simulation result without the external magnetic field with the result with $B_{0}$. The physical quantities related to $B_{0}$ like the ratio of $\omega_{c e}$ to $\omega_{p e}$, plasma beta $\beta$, Larmor radius $r_{L}$, and Alfvén velocity $V_{A}$ are $2.0,0.005,0.5 \Delta$, and $0.81 \mathrm{c}$, respectively.

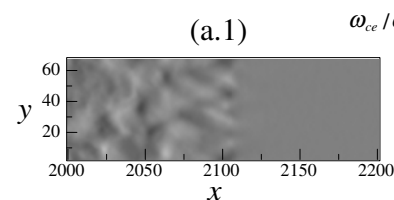

$\omega_{c e} / \omega_{p e}=0$

(b.1)

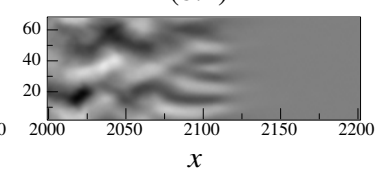

(a.2)

$\omega_{c e} / \omega_{p e}=2$.

(b.2)
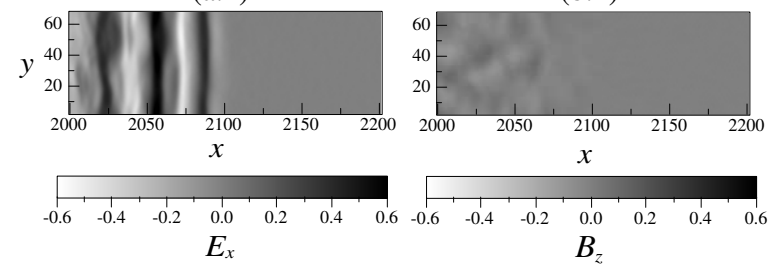

Figure 2: The spatial distribution of the generated electric fields, $E_{x}$ (a.1-2) and $B_{z}$ (b.1-2) at $\omega_{p e} t=25$. The plotted region is a part of right side of the system, where the position $2000 \Delta$ is the center of the system.

\section{Simulation Results}

The both electric field and magnetic field are generated by counterstreaming instability. In figure 2 , (a.1) and (b.1) show the spatial distribution of generated electric field $E_{x}$ which has the component parallel to the streaming direction, and generated magnetic field $B_{z}$ which has the component normal to the streaming direction, respectively, at $\omega_{p e} t=25$, without the external magnetic field parallel to the streaming direction. (a.2) and (b.2) show the spatial distribution of generated electric field $E_{x}$ and generated magnetic field $B_{z}$, respectively, at $\omega_{p e} t=25$, when $\omega_{c e} / \omega_{p e}=2$. As seen in Fig.2 (b.1) and (b.2), the generation of magnetic fields due to the electromagnetic counterstreaming instability (EMCSI) is restrained by the external magnetic field, while as seen in Fig.2 (b.1) and (b.2), the generation of electrostatic fields due to the electrostatic counterstreaming instability (ES-CSI) is enhanced. The results show that the counterstreaming instability changes its character from magnetic to electrostatic nature when the external magnetic field increases. The generated electrostatic field can accelerates strongly the particles up to the relativistic velocity in the nonlinear phase.

Figure 3 shows the time development of electric field $E_{x}$ on a part of the right side region at (a) $\omega_{p e} t=25$, (b) $\omega_{p e} t=75$, (c) $\omega_{p e} t=125$ when $\omega_{c e} / \omega_{p e}=2$. The dashed lines show the estimated front of streaming plasma with the velocity $0.5 \mathrm{c}$ at each times. In Fig.3(a), the electrostatic waves are generated within the region where the counterstreaming plasmas are overlapped. However as seen in Fig.3(c), the electrostatic waves are generated beyond the front. This means the production 


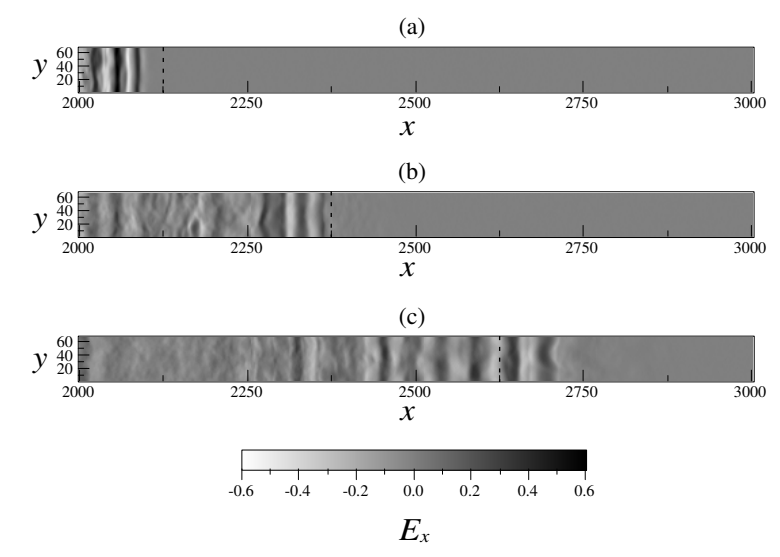

Figure 3: Time development of electric field $E_{x}$ on a part of the right side region at (a) $\omega_{p e} t=25$, (b) $\omega_{p e} t=$ 75 , (c) $\omega_{p e} t=125$ when $\omega_{c e} / \omega_{p e}=2$. The dashed lines show the estimated front of streaming plasma with the velocity $0.5 \mathrm{c}$ at each times.

of particles which are moving faster than the initial plasma streaming velocity to generate the electrostatic waves beyond it.

Finally, we present the electron energy distribution in Fig. 4. The horizontal axis shows the electron Lorentz $\Gamma$, while the vertical axis shows $\log f_{e}$. The dotted and solid distributions are the initial and final state $\left(\omega_{p e} t=150\right)$, respectively. As seen in Figs. 4(a) and $4(\mathrm{~b})$, the particles are more effectively accelerated by the electrostatic waves under the external magnetic field. The energy spectra of both conditions in their high energy regions are characterized by an exponential-type law. From these simulations we conclude that both electrons and positrons can be accelerated with $\mathrm{MeV}$ energy during the counterstreaming instability. For the strong external magnetic field, the particle acceleration in pair plasmas becomes more effective than for the case of unmagnetized pair plasmas.

\section{Summary and Discussions}

We investigated the electromagnetic counterstreaming instability in magnetized pair plasmas, using a two-dimensional, fully electromagnetic, relativistic particle-in-cell code. It was found from the simulations that the generated electrostatic waves can accelerate both electrons and positrons up to the energy of a few $M e V$. We found that the energy spectrum of the accelerated particles is the exponentialtype in the high energy region.

The authors [12] [13] [14] presented the counterstreaming instability by using the three dimen-
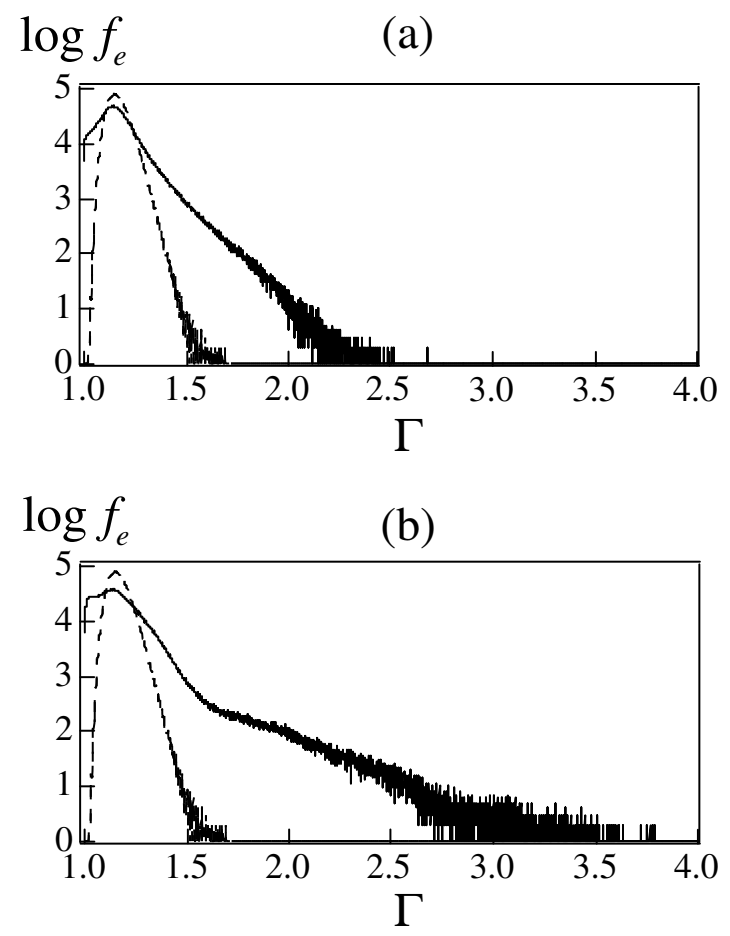

Figure 4: The electron energy distributions in the condition (a) $\omega_{c e} / \omega_{p e}=0$ and (b) $\omega_{c e} / \omega_{p e}=2$. The horizontal axis shows the electron Lorentz $\Gamma$, while the vertical axis shows $\log f_{e}$. The dotted and solid distributions are the initial and final state $\left(\omega_{p e} t=150\right)$, respectively.

sional particle-in-cell code. They showed that the generated quasi-static magnetic fields evolve from the short-scale to long-scale magnetic field. Only Nishikawa et al.[12] investigated the effects of weak external magnetic field to counterstreaming instability. Their result was similar to those without the external magnetic field. However, if there is the strong, ordered magnetic field that can restrain the instability from generation of magnetic field, the counterstreaming instability may begin to change from electromagnetic to the electrostatic nature. Thus, the small scale magnetic field, i.e. the current filaments along the plasma flows can't be well evolved. Therefore, the perpendicular heating due to the scattering of particles by generated magnetic fields tend to be suppressed. As the results, the kinetic energy of plasma flows may be effectively converted to the energy of electrostatic field that can well accelerate the particles in the direction parallel to the background magnetic field. The high-energy particles acceleration due to electrostatic field becomes more efficient contrast to the case of the parallel acceleration obtained by the authors [12] [13] [14].

The origin of energetic GRBs is still unknown. But the detection of polarization of the gamma-rays 
by Coburn and Boggs[5] provides new insight into the mechanism driving the GRBs. From earlier observations of the gamma-ray spectrum of GRBs, it was concluded that the most likely mechanism for GRBs is synchrotron emission. That Coburn and Boggs detect a clear polarization in the gammarays from a burst provides direct evidence in support of synchrotron emission as the mechanism of GRBs. From polarization produced by the synchrotron mechanism, the gamma-ray producing region is suffused by an ordered magnetic field, oriented in the same direction.

The counterstreaming instability in magnetized pair plasmas may be applicable for the GRBs mechanism for both production of collisionless shocks as well as high energy particles of order of a few $M e V$. However, since there is only single observation that proves the existence of ordered magnetic field at GRBs source, we need the more observational evidence which demonstrates the presence of strong, ordered magnetic field.

\section{References}

[1] P. Meszaros, Ann. Rev. Astron. Astrophys. 40, 137 (2002)

[2] T. Piran, Phys. Rep. 333, 529 (2000)

[3] M. V. Medvedev, and A. Loeb, Astrophys. J. 526, 697 (1999)

[4] E. W. Weibel, Phys. Rev. Lett. 2, 83 (1959)

[5] W. Coburn and S. E. Boggs, Nature, 423, 415 (2003)

[6] F. Califano, F. Pegoraro, and S. V. Bulanov, Phys. Rev. E 56, 963 (1997)

[7] M. Honda, J. Meyer-ter-Vehn, and A. M. Pukov, Phys. Rev. Lett. 85, 2128 (2000)

[8] J. I. Sakai, S. Saito, H. Mae, D. Farina, M. Lontano, F. Califano, F. Pegoraro, and S. V. Bulanov, Phys. Plasmas 9, 2959 (2002)

[9] S. Saito, and J. I. Sakai, J. Phys. Soc. Jpn. 71, 1931, (2002)

[10] Y. Kazimura, J. I. Sakai, T. Neubert, and S. V. Bulanov, Astrophys. J. Lett. 498, L183 (1998)

[11] T. Haruki, and J. I. Sakai, Phys. Plasma 10, $392(2003)$

[12] K. I. Nishikawa, P. Hardee, G. Richardson, R. Preece, H. Sol, and G. J. Fishman, Astrophys. J. 595, 555 (2003)
[13] L. O. Silva, R. A. Fonseca, J. W. Tonge, J. M. Dawson, W. B. Mori, and M. V. Medvedev, Astrophys. J. Lett. 596, L121 (2003)

[14] R. A. Fonseca, L. O. Silva, J. W. Tonge, W. B. Mori, and J. M. Dawson, Phys. Plasma 10, 1979 (2003)

[15] O. Buneman, Computer Space Plasma Physics, Simulation Techniques and Software (Terra Scientific, New York, 1993) 